\title{
Facile Chlorination of Benzyl Alcohols Using 1,8-Diazabicyclo[5.4.0]undec-7-ene (DBU) and Sulfonyl Chlorides
}

\author{
Hyung Woo Kim, ${ }^{\dagger, t}$ Yun-Sang Lee, ${ }^{\dagger, *}$ Dinesh Shetty, ${ }^{\dagger}$ Hak Jeong Lee, ${ }^{\dagger}$ Dong Soo Lee, ${ }^{\dagger}$ June-Key Chung, ${ }^{\dagger}$ \\ Myung Chul Lee, ${ }^{\dagger}$ Kyoo-Hyun Chung, ${ }^{\S}$ and Jae Min Jeong ${ }^{\dagger, *}$
}

\begin{abstract}
${ }^{\dagger}$ Department of Nuclear Medicine, Cancer Research Institute, Seoul National University College of Medicine, 28 Yungundong Chongrogu, Seoul 110-744, Korea.*E-mail:jmjng@snu.ac.kr(J.M.J),wonza43@snu.ac.kr (Y.-S.L.) ${ }^{\ddagger}$ Department of Testing and Research, Korea Consumer Agency, 108 Yangjaedong Seochogu, Seoul 137-700, Korea ${ }^{\S}$ Department of Chemistry, Inha University, 253 Yonghyundong Namgu, Inchon 402-751, Korea
\end{abstract} Received June 4, 2010, Accepted September 10, 2010

Key Words: Chlorination, Benzyl alcohol, DBU, Toluenesulfonyl chloride, Methanesulfonyl chloride

Chlorinated compounds are useful intermediates in many organic synthetic reactions. A variety of biologically active natural products have chlorine atoms in their structure. Thus, chlorination of alcohols has been studied and used in organic chemistry for decades. Chlorination of benzyl alcohols is also a topic of interest because benzyl chlorides can be used as important synthons in organic synthesis. Generally, chlorination of alcohols has been performed using traditional reagents such as concentrated $\mathrm{HCl},{ }^{1} \mathrm{SOCl}_{2},{ }^{2} \mathrm{PCl}_{5},{ }^{3} \mathrm{PCl}_{3},{ }^{4}$ and $\mathrm{POCl}_{3} .{ }^{5}$ However, these reagents are strongly acidic and corrosive, and often require heating for the reaction. Many attempts have been made to develop non-acidic and mild chlorination conditions. Benzyl alcohols can be chlorinated by traditional methods; however, milder and easier reaction conditions are necessary for an increased yield. Chlorination using $\mathrm{PPh}_{3} / \mathrm{CCl}_{4}{ }^{6}$ or a modifiedMitsunobu type reaction ${ }^{7}$ are relatively mild reactions. Chlorination by silyl chlorides with catalysts might be another option. ${ }^{8-11}$ In case of benzyl alcohols, chlorination using sulfonyl chlorides such as methanesulfonyl chloride $(\mathrm{MsCl})$ or $p$-toluenesulfonyl chloride $(\mathrm{TsCl})$ with organic bases also could be of choice. ${ }^{12-18}$ In the present study, 1,8-diazabicyclo[5.4.0]undec-7-ene (DBU), one of the strongest organic bases, was tested as an alternative choice for chlorination of benzyl alcohols.

We estimated chlorination yields of 4-bromobenzyl alcohol using various amounts of sulfonylating agents and DBU from $0{ }^{\circ} \mathrm{C}$ to room temperature for $1 \mathrm{hr}$ (Table 1 ). The TLC monitoring showed that chlorination occurred after sulfonylation. Long reaction time was not necessary because the completion of reaction occurred during concentration process of the reaction mixture. The reaction gave 4-bromobenzyl chloride in low yields when reacted with 1 eq. of each reagent (entry 1 and 6). The yields were improved to $83 \%$ with $\mathrm{TsCl}$ and $92 \%$ with $\mathrm{MsCl}$ as the amount of added reagents increased (entry 4 and 10). The reaction with 1 eq. of TsCl gave 4-bromobenzyl toluenesulfonate in low yield (entry 1). No methanesulfonate was separated in the trials with $\mathrm{MsCl}$ (entry 6-10). To test the substituent effect of the aromatic ring, chlorination of three benzyl alcohols containing either $-\mathrm{NO}_{2},-\mathrm{H}$ or $-\mathrm{OCH}_{3}$ at the 4-position were carried out with 2.0 eq. DBU and 1.5 eq. $\mathrm{TsCl}$ or $\mathrm{MsCl}$ (Table 2). With $\mathrm{TsCl}$, the yields decreased according to the increase of electron density on the aromatic ring (entry 1,3 and 5), whereas
Table 1. Chlorination test of 4-bromobenzyl alcohol according to the amounts of DBU and sulfonylating agents ${ }^{a}$

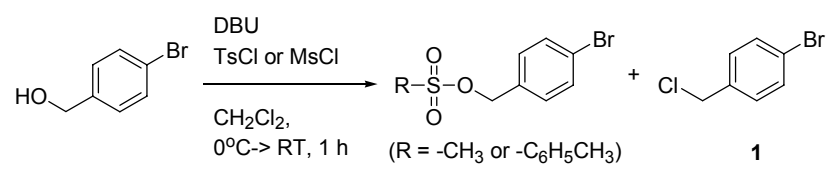

\begin{tabular}{|c|c|c|c|c|}
\hline \multirow{2}{*}{ Entry } & \multirow{2}{*}{ DBU (eq.) } & \multirow{2}{*}{$\begin{array}{c}\text { Sulfonyl } \\
\text { chloride (eq.) }\end{array}$} & \multicolumn{2}{|c|}{ Yield $(\%)^{b}$} \\
\hline & & & Sulfonylate & Chloride 1 \\
\hline 1 & 1.0 & $\mathrm{TsCl}(1.0)$ & 5 & 48 \\
\hline 2 & 1.5 & $\mathrm{TsCl}(1.5)$ & - & 60 \\
\hline 3 & 2.0 & $\mathrm{TsCl}(1.5)$ & - & 66 \\
\hline 4 & 2.0 & $\mathrm{TsCl}(2.0)$ & - & 83 \\
\hline 5 & 3.0 & $\mathrm{TsCl}(3.0)$ & - & 74 \\
\hline 6 & 1.0 & $\mathrm{MsCl}(1.0)$ & - & 55 \\
\hline 7 & 1.5 & $\mathrm{MsCl}(1.5)$ & - & 64 \\
\hline 8 & 2.0 & $\mathrm{MsCl}(1.5)$ & - & 69 \\
\hline 9 & 2.0 & $\mathrm{MsCl}(2.0)$ & - & 77 \\
\hline 10 & 3.0 & $\mathrm{MsCl}(3.0)$ & - & 92 \\
\hline
\end{tabular}

${ }^{a}$ 4-Bromobenzyl alcohol $(1.32-1.36 \mathrm{mmol})$ was used with $10-15 \mathrm{~mL}$ $\mathrm{CH}_{2} \mathrm{Cl}_{2} .{ }^{b}$ Isolated yields.

Table 2. Chlorination of three benzyl alcohols using DBU and sulfonylating agents ${ }^{a}$

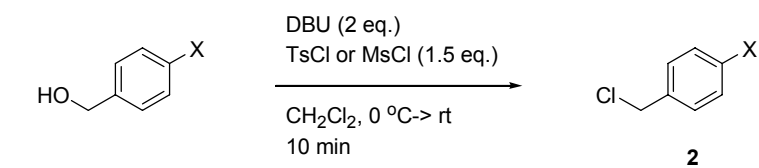

\begin{tabular}{ccccccc}
\hline Entry & $\mathrm{X}$ & \multirow{2}{*}{$\begin{array}{c}\text { Sulfornyl } \\
\text { chloride }\end{array}$} & Chloride & $\begin{array}{r}\text { Yield } \\
(\%)^{b}\end{array}$ & \multicolumn{2}{c}{$\delta$ of Benzylic H } \\
\cline { 6 - 7 } & & & & & Alcohol & Chloride 2 \\
\hline 1 & $\mathrm{NO}_{2}$ & $\mathrm{TsCl}$ & $\mathbf{2 a}$ & 81 & 4.84 & 4.66 \\
2 & $\mathrm{NO}_{2}$ & $\mathrm{MsCl}$ & $\mathbf{2 a}$ & 69 & & \\
3 & $\mathrm{H}$ & $\mathrm{TsCl}$ & $\mathbf{2 b}$ & 59 & 4.63 & 4.55 \\
4 & $\mathrm{H}$ & $\mathrm{MsCl}$ & $\mathbf{2 b}$ & 65 & & \\
5 & $\mathrm{OMe}$ & $\mathrm{TsCl}$ & $\mathbf{2 c}$ & 44 & 4.60 & 4.57 \\
6 & $\mathrm{OMe}$ & $\mathrm{MsCl}$ & $\mathbf{2 c}$ & 79 & & \\
\hline
\end{tabular}

${ }^{a}$ Benzyl alcohols (1.32 - $1.36 \mathrm{mmol}$ ) were used with $10-15 \mathrm{~mL} \mathrm{CH}_{2} \mathrm{Cl}_{2}$. ${ }^{b}$ Isolated yields. 
Table 3. Chlorination of benzyl alcohols using DBU and sulfonylating agents ${ }^{a}$

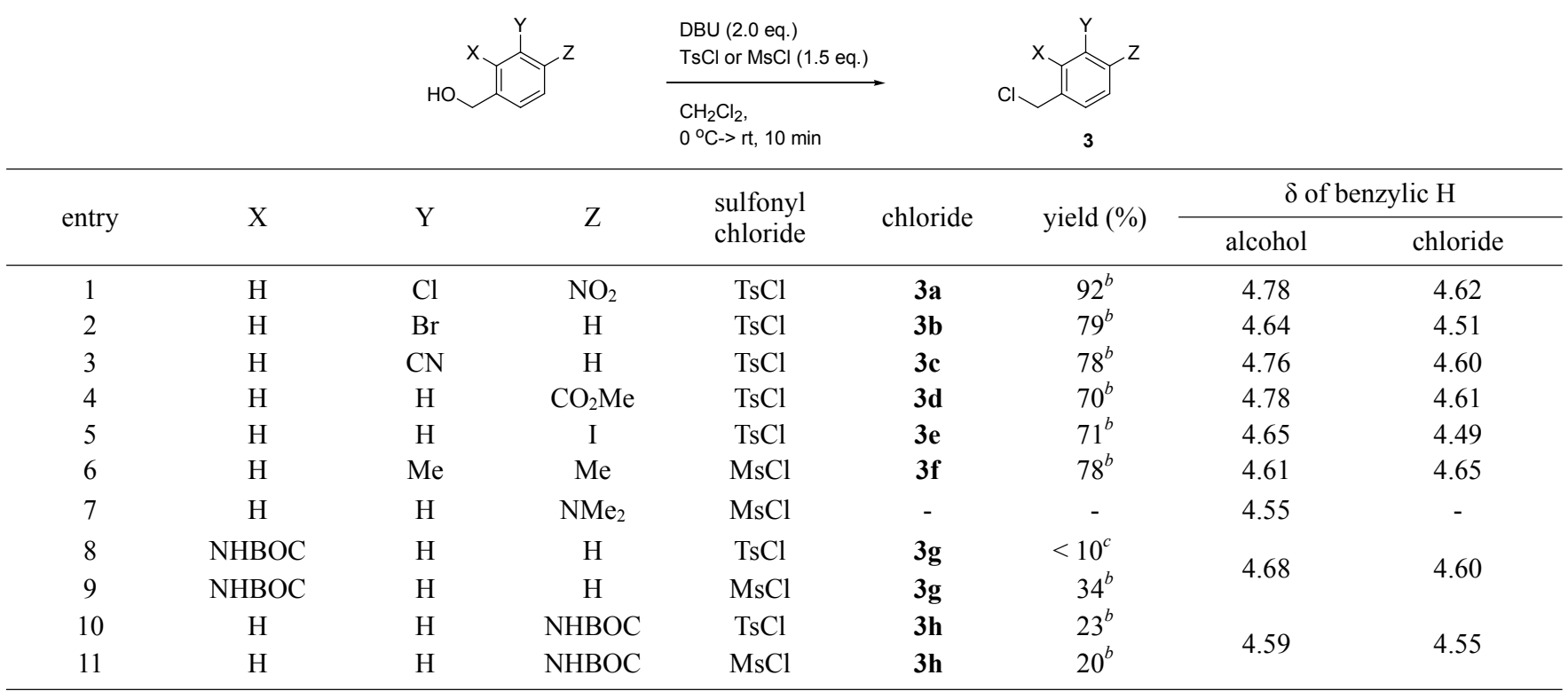

${ }^{\bar{a} \text { Benzyl alcohols (1.32 - } 1.36 \mathrm{mmol} \text { ) were used with } 10-15 \mathrm{~mL} \mathrm{CH}_{2} \mathrm{Cl}_{2} \text { (entry a-g) or } 1-5.5 \mathrm{~mL} \text { (entry h). }{ }^{b} \text { Isolated yields. }{ }^{c} \text { Assumed from }{ }^{1} \mathrm{H} \text { NMR }}$ of inseparable mixture ( $3 \mathrm{~g}$ and $\mathrm{TsCl})$ after column purification.

the yields were not significantly affected by electron density on the aromatic ring in case of $\mathrm{MsCl}$ (entry 2, 4 and 6). Further tests were carried out based on the results in Table 2: Electronwithdrawing group (EWG) containing benzyl alcohols were tested with $\mathrm{TsCl}$ and electron-donating group (EDG) containing benzyl alcohols were tested with $\mathrm{MsCl}$ (Table 3). In reactions with the EWG-containing benzyl alcohols, the benzyl chlorides were synthesized with increased yields (entry 1-5). On the other hand, the reactions of the EDG-containing benzyl alcohols did not always give increased yields. The 3,4-dimethylbenzyl alcohol gave its corresponding chloride in a moderate yield (entry 6). However, the 4- $N, N$-dimethylaminobenzyl alcohol did not give a chlorinated product but gave a polar mixture at the origin on TLC (entry 7). All of the reactions containing BOCprotected aminobenzyl alcohols, at the ortho or para position of the benzyl alcohols, gave chlorinated products in low yields (entry 8-11). The mechanism of this reaction is supposed to be sulfonylation followed by substitution of chloride. In case of EWG-containing benzyl alcohol, tosylates give good yields because they are relatively stable and also are appropriately reactive comparing with their mesylates. In case of EDG-containing benzyl alcohols, their tosylates might be too stable to react with chloride comparing with the mesylates. In this study, various benzyl chloride derivatives were prepared with good to moderate yields in a short time under ambient condition. Although there are many reports of chlorination using sulfonyl chlorides and bases (like TEA and DMAP), short reaction time and easy reaction condition of this procedure could be advantages for some cases. For an example, chlorination of electrondeficient benzyl alcohols often requires harsher conditions and longer reaction times than the present method: Preparation of 4-nitrobenzyl chloride from its corresponding alcohol necessitates very long time $(24 h)^{11,19}$ or a high temperature $(100-150$ $\left.{ }^{\circ} \mathrm{C}\right){ }^{20,21}$ However, 4-nitrobenzyl alcohol was chlorinated within
10 min with $81 \%$ yield using DBU and $\mathrm{TsCl}$ in this study. A facile and fast method for chlorination of benzyl alcohols was developed in this study. This method is not effective for aminecontaining substrates, possibly because of side reaction by free amino group or because of electron-donating property of amino group on ortho- or para- postion by resonance. But in some cases, for example electron deficient benzyl alcohols, this method could be a choice for chlorination.

\section{Experimental Section}

General procedure for preparation of benzyl chlorides. To a mixture of benzyl alcohol ( $1.34 \mathrm{mmol})$ in $10.0 \mathrm{~mL} \mathrm{CH}_{2} \mathrm{Cl}_{2}, \mathrm{DBU}$ $(2.66 \mathrm{mmol})$ and $\mathrm{TsCl}(2.06 \mathrm{mmol})$ in $5.0 \mathrm{~mL} \mathrm{CH}_{2} \mathrm{Cl}_{2}$ or neat $\mathrm{MsCl}(2.06 \mathrm{mmol})$ were added in an ice-water bath. After the compounds were added, the bath was removed and the mixture was stirred at room temperature. After $10 \mathrm{~min}$, the mixture was concentrated under reduced pressure (bath temperature: 31 $\left.32{ }^{\circ} \mathrm{C}\right)$. Column chromatography on silica gel $(230$ - 400 mesh, mobile phase: 5 - $40 \%$ EtOAc/Hexane.

4-Bromobenzyl chloride 1: white solid ( $66 \%$ for $\mathrm{TsCl}$ and $69 \%$ for $\mathrm{MsCl}$ ); mp $35-36{ }^{\circ} \mathrm{C}$ (lit. $\left.37-38{ }^{\circ} \mathrm{C}\right) ;{ }^{1} \mathrm{H}$ NMR (300 $\left.\mathrm{MHz}, \mathrm{CDCl}_{3}\right) \delta 7.46(\mathrm{~d}, J=8.4 \mathrm{~Hz}, 2 \mathrm{H}), 7.23(\mathrm{~d}, J=8.4 \mathrm{~Hz}$, $2 \mathrm{H}), 4.50(\mathrm{~s}, 2 \mathrm{H}){ }^{13} \mathrm{C}$ NMR $\left(75 \mathrm{MHz}, \mathrm{CDCl}_{3}\right) \delta 136.3(\mathrm{C})$, 131.8 (CH) $130.2(\mathrm{CH}) 122.4(\mathrm{C}), 45.3\left(\mathrm{CH}_{2}\right) ; \mathrm{MS}(\mathrm{CI}+): \mathrm{m} / \mathrm{z}$ $205(\mathrm{M}+1)$.

4-Nitrobenzyl chloride 2a: white solid ( $81 \%$ for $\mathrm{TsCl}$ and $69 \%$ for $\mathrm{MsCl}$ ); mp $70-71{ }^{\circ} \mathrm{C}$ (lit. $\left.70-73{ }^{\circ} \mathrm{C}\right) ;{ }^{1} \mathrm{H}$ NMR $(300$ $\left.\mathrm{MHz}, \mathrm{CDCl}_{3}\right) \delta 8.21(\mathrm{~d}, J=8.4 \mathrm{~Hz}, 2 \mathrm{H}), 7.58(\mathrm{~d}, J=8.7 \mathrm{~Hz}$, $2 \mathrm{H}), 4.66(\mathrm{~s}, 2 \mathrm{H}) ;{ }^{13} \mathrm{C}$ NMR $\left(75 \mathrm{MHz}, \mathrm{CDCl}_{3}\right) \delta 147.5(\mathrm{C})$, $144.2(\mathrm{C}) 129.2(\mathrm{CH}) 123.8(\mathrm{CH}), 44.4\left(\mathrm{CH}_{2}\right) ; \mathrm{MS}(\mathrm{CI}+) \mathrm{m} / \mathrm{z}$ $171(\mathrm{M}+1)$.

Benzyl chloride 2b: clear oil (59\% for $\mathrm{TsCl}$ and $65 \%$ for $\mathrm{MsCl}) ;{ }^{1} \mathrm{H}$ NMR $\left(300 \mathrm{MHz}, \mathrm{CDCl}_{3}\right) \delta$ 7.35-7.29 (m, 5H), 4.55 
$(\mathrm{s}, 2 \mathrm{H}) ;{ }^{13} \mathrm{C} \mathrm{NMR}\left(75 \mathrm{MHz}, \mathrm{CDCl}_{3}\right) \delta 137.4(\mathrm{C}) 128.6(\mathrm{CH})$ $128.5(\mathrm{CH}), 128.3(\mathrm{CH}), 46.2\left(\mathrm{CH}_{2}\right)$; $\mathrm{MS}(\mathrm{CI}+) \mathrm{m} / z 127(\mathrm{M}+1)$.

4-Methoxybenzyl chloride 2c: clear oil (44\% for $\mathrm{TsCl}$ and $79 \%$ for $\mathrm{MsCl}$ ); ${ }^{1} \mathrm{H} \mathrm{NMR}\left(300 \mathrm{MHz}, \mathrm{CDCl}_{3}\right) \delta 7.31$ (d, $J=8.4$ $\mathrm{Hz}, 2 \mathrm{H}), 6.88$ (d, $J=8.4 \mathrm{~Hz}, 2 \mathrm{H}), 4.57$ (s, 2H), 3.80 (s, 3H);

${ }^{13} \mathrm{C} \mathrm{NMR}\left(75 \mathrm{MHz}, \mathrm{CDCl}_{3}\right) \delta 159.6(\mathrm{C}), 130.0(\mathrm{C}) 129.6(\mathrm{CH})$ $114.0(\mathrm{C}), 55.2\left(\mathrm{CH}_{3}\right), 46.2\left(\mathrm{CH}_{2}\right)$; $\mathrm{MS}(\mathrm{CI}+) \mathrm{m} / z 157(\mathrm{M}+1)$.

1-Chloro-4-(chloromethyl)-2-nitrobenzene 3a: yellow oil (92\% for TsCl); ${ }^{1} \mathrm{H}$ NMR $\left(300 \mathrm{MHz}, \mathrm{CDCl}_{3}\right) \delta 7.92(\mathrm{~s}, 1 \mathrm{H})$, $7.56(\mathrm{~m}, 2 \mathrm{H}), 4.62(\mathrm{~s}, 2 \mathrm{H}) ;{ }^{13} \mathrm{C} \mathrm{NMR}\left(75 \mathrm{MHz}, \mathrm{CDCl}_{3}\right) \delta 147.6$ (C), $137.7(\mathrm{C}), 133.0(\mathrm{CH}) 132.1(\mathrm{CH}), 126.7(\mathrm{C}), 125.4(\mathrm{CH})$, $43.8\left(\mathrm{CH}_{2}\right)$; MS (EI+) $m / z 205\left(\mathrm{M}^{+}, 70\right)$.

3-Bromobenzyl chloride 3b: clear oil (79\% for $\mathrm{TsCl})$; ${ }^{1} \mathrm{H}$ NMR $\left(300 \mathrm{MHz}, \mathrm{CDCl}_{3}\right) \delta 7.53(\mathrm{~s}, 1 \mathrm{H}), 7.43(\mathrm{~d}, J=8.1 \mathrm{~Hz}, 1 \mathrm{H})$, $7.29(\mathrm{~d}, J=7.8 \mathrm{~Hz}, 1 \mathrm{H}), 7.20(\mathrm{dd}, J=8.1,7.8 \mathrm{~Hz}, 1 \mathrm{H}), 4.51(\mathrm{~s}$, $2 \mathrm{H}) ;{ }^{13} \mathrm{C}$ NMR $\left(75 \mathrm{MHz}, \mathrm{CDCl}_{3}\right) \delta 139.5(\mathrm{C}), 131.5(\mathrm{CH}), 131.4$ (CH) $130.2(\mathrm{CH}), 127.1(\mathrm{CH}), 122.5(\mathrm{C}), 45.1\left(\mathrm{CH}_{2}\right)$; MS (EI+) $m / z 204\left(\mathrm{M}^{+}\right)$.

3-(Chloromethyl)benzonitrile 3c: white solid (78\% for $\mathrm{TsCl}$ ); mp 64 - $66{ }^{\circ} \mathrm{C}$ (lit. $\left.65-67{ }^{\circ} \mathrm{C}\right) ;{ }^{1} \mathrm{H}$ NMR $\left(300 \mathrm{MHz}, \mathrm{CDCl}_{3}\right) \delta$ $7.68(\mathrm{~s}, 1 \mathrm{H}), 7.65-7.59(\mathrm{~m}, 2 \mathrm{H}), 7.46(\mathrm{dd}, J=7.8,7.5,1 \mathrm{H}), 4.60$ $(\mathrm{s}, 2 \mathrm{H}) ;{ }^{13} \mathrm{C}$ NMR $\left(75 \mathrm{MHz}, \mathrm{CDCl}_{3}\right) \delta 138.6(\mathrm{C}), 132.7(\mathrm{CH})$, $131.72(\mathrm{CH}), 131.70(\mathrm{CH}), 129.4(\mathrm{CH}), 118.0(\mathrm{C}), 112.5(\mathrm{C})$, $44.5\left(\mathrm{CH}_{2}\right)$; MS (EI+) $m / z 151\left(\mathrm{M}^{+}\right)$.

Methyl 4-(chloromethyl)benzoate 3d: white solid (70\% for $\mathrm{TsCl}$ ); mp 37 - $38{ }^{\circ} \mathrm{C}$ (lit. $\left.40{ }^{\circ} \mathrm{C}\right) ;{ }^{1} \mathrm{H}$ NMR (300 MHz, $\mathrm{CDCl}_{3}$ ) $\delta 8.03(\mathrm{~d}, J=8.4 \mathrm{~Hz}, 2 \mathrm{H}), 7.45(\mathrm{~d}, J=8.1 \mathrm{~Hz}, 2 \mathrm{H}), 4.61(\mathrm{~s}, 2 \mathrm{H})$, $3.92(\mathrm{~s}, 3 \mathrm{H}) ;{ }^{13} \mathrm{C} \mathrm{NMR}\left(75 \mathrm{MHz}, \mathrm{CDCl}_{3}\right) \delta 166.5(\mathrm{C}), 142.2(\mathrm{C})$, $130.0(\mathrm{CH}) 129.9(\mathrm{CH}), 128.4(\mathrm{CH}), 52.1\left(\mathrm{CH}_{3}\right), 45.3\left(\mathrm{CH}_{2}\right)$; MS (EI+) $m / z(\%) 184\left(\mathrm{M}^{+}\right)$.

4-Iodobenzyl chloride 3e: white solid (71\% for $\mathrm{TsCl})$; $\mathrm{mp}$ 48 - $50{ }^{\circ} \mathrm{C}$ (lit. $\left.52-53{ }^{\circ} \mathrm{C}\right) ;{ }^{1} \mathrm{H}$ NMR $\left(300 \mathrm{MHz}, \mathrm{CDCl}_{3}\right) \delta 7.66$ $(\mathrm{d}, J=8.4 \mathrm{~Hz}, 2 \mathrm{H}), 7.10(\mathrm{~d}, J=8.4 \mathrm{~Hz}, 2 \mathrm{H}), 4.49(\mathrm{~s}, 2 \mathrm{H}) ;{ }^{13} \mathrm{C}$ NMR $\left(75 \mathrm{MHz}, \mathrm{CDCl}_{3}\right) \delta 137.8(\mathrm{CH}), 137.0(\mathrm{C}), 130.3(\mathrm{CH})$, 94.1 (C) $45.4\left(\mathrm{CH}_{2}\right)$; MS (CI+) $m / z 253(\mathrm{M}+1)$.

4-(Chloromethyl)-1,2-dimethylbenzene 3f: clear oil (78\% for $\mathrm{MsCl}) ;{ }^{1} \mathrm{H} \mathrm{NMR}\left(300 \mathrm{MHz}, \mathrm{CDCl}_{3}\right) \delta 7.28(\mathrm{~s}, 1 \mathrm{H}), 7.24(\mathrm{~s}$, 2H), 4.65 (s, 2H), $2.38(\mathrm{~s}, 6 \mathrm{H}) ;{ }^{13} \mathrm{C} \mathrm{NMR}\left(75 \mathrm{MHz}, \mathrm{CDCl}_{3}\right) \delta$ 136.9 (C), $136.8(\mathrm{C}), 134.8(\mathrm{C}), 129.84(\mathrm{CH}), 129.79(\mathrm{CH})$, $126.0(\mathrm{CH}), 46.2\left(\mathrm{CH}_{2}\right), 19.6\left(\mathrm{CH}_{3}\right), 19.4\left(\mathrm{CH}_{3}\right) ; \mathrm{MS}(\mathrm{EI}+) \mathrm{m} / \mathrm{z}$ $154\left(\mathrm{M}^{+}\right)$.

tert-Butyl 2-(chloromethyl)phenylcarbamate 3g: white solid (less then $10 \%$ for $\mathrm{TsCl}$ and $34 \%$ for $\mathrm{MsCl}$ ); $\mathrm{mp} 47-48{ }^{\circ} \mathrm{C} ;{ }^{1} \mathrm{H}$ NMR (300 MHz, $\left.\mathrm{CDCl}_{3}\right) \delta 7.84(\mathrm{~d}, J=8.1 \mathrm{~Hz}, 1 \mathrm{H}), 7.34$ (dd, $J=8.1,7.5 \mathrm{~Hz}, 2 \mathrm{H}), 7.26(\mathrm{~d}, J=7.2 \mathrm{~Hz}, 1 \mathrm{H}), 7.06(\mathrm{dd}, J=7.5$, $7.2 \mathrm{~Hz}, 1 \mathrm{H}), 6.77(\mathrm{bs}, 1 \mathrm{H}), 4.60(\mathrm{~s}, 2 \mathrm{H}), 1.53(\mathrm{~s}, 9 \mathrm{H}) ;{ }^{13} \mathrm{C} \mathrm{NMR}$ $\left(75 \mathrm{MHz}, \mathrm{CDCl}_{3}\right) \delta 152.9(\mathrm{C}), 137.0(\mathrm{C}), 130.0(\mathrm{CH}), 127.0(\mathrm{C})$,
$123.9(\mathrm{CH}), 122.7(\mathrm{CH}), 80.8(\mathrm{C}), 44.1\left(\mathrm{CH}_{2}\right), 28.2\left(\mathrm{CH}_{3}\right)$; $\mathrm{MS}$ (EI+) $m / z 241\left(\mathrm{M}^{+}\right)$.

tert-Butyl 4-(chloromethyl)phenylcarbamate $3 \mathrm{~h}$ : white solid (23\% for $\mathrm{TsCl}$ and $20 \%$ for $\mathrm{MsCl}$ ); mp $92-94{ }^{\circ} \mathrm{C} ;{ }^{1} \mathrm{H} \mathrm{NMR}$ $\left(300 \mathrm{MHz}, \mathrm{CDCl}_{3}\right) \delta 7.35(\mathrm{~d}, J=8.4 \mathrm{~Hz}, 2 \mathrm{H}), 7.30(\mathrm{~d}, J=8.4$ $\mathrm{Hz}, 2 \mathrm{H}), 6.58(\mathrm{bs}, 1 \mathrm{H}), 4.55(\mathrm{~s}, 2 \mathrm{H}), 1.52(\mathrm{~s}, 9 \mathrm{H}) ;{ }^{13} \mathrm{C} \mathrm{NMR}$ $\left(75 \mathrm{MHz}, \mathrm{CDCl}_{3}\right) \delta 152.6(\mathrm{C}), 138.5(\mathrm{C}), 131.9(\mathrm{C}), 129.4(\mathrm{CH})$, $118.5(\mathrm{CH}), 80.7(\mathrm{C}), 46.1\left(\mathrm{CH}_{2}\right), 28.3\left(\mathrm{CH}_{3}\right)$; MS (EI+) $m / z 241$ $\left(\mathrm{M}^{+}\right)$.

Acknowledgments. This work was supported by National Research Foundation of Korea funded by Ministry of Education, Science and Technology (R0A-2008-000-20116-0 and 2010-K001055).

\section{References}

1. Norris, J. F.; Watt, M.; Thomas, R. J. Am. Chem. Soc. 1916, 38, 1071

2. Gilman, H.; Kirby, J. E. J. Am. Chem. Soc. 1929, 51, 3475.

3. Burks, J. E., Jr. Phosphorus(V) Chloride In Encyclopedia of Reagents for Organic Synthesis; Paquette, L., Ed.; 2004; Wiley \& Sons: New York.

4. Anderson, A. G., Jr.; Owen, N. E. T.; Freenor, F. J.; Erickson, D. Synthesis 1976, 398.

5. Tipson, R. S. J. Org. Chem. 1962, 27, 1449.

6. Appel, R. Angew. Chem. Intl. Ed. 1975, 14, 801.

7. Mitsunobu, O. Synthesis 1981, 1.

8. Lee, J. G.; Kang K. K. J. Org. Chem. 1988, 53, 3634.

9. Ha, D. S.; Chai, G. S. Bull. Korean Chem. Soc. 1997, 8, 1045.

10. Ha, D. S.; Yoon, M. J. J. Korean Chem. Soc. 1997, 41, 541.

11. Yasuda, M.; Yamasaki, S.; Onishi, Y.; Baba, A. J. Am. Chem. Soc. 2004, 126, 7186-7187.

12. Pearson, W. H.; Walavalkar, R. Tetrahedron 1994, 50, 1229312304.

13. Larock, R. C.; Babu, S. Tetrahedron 1987, 43, 2013-2020.

14. Douty, B. D.; Salvmo, J. M.; Seoane, P. R.; Dolle, R. E. Bioorg. Med. Chem. Lett. 1995, 5, 363-366.

15. Ballester, M.; Veciana, J.; Riera, J.; Castaiier, J.; Rovira, C.; Armet, O. J. Org. Chem. 1986, 51, 2472-2480.

16. Armaroli, N.; Barigelletti, F.; Ceroni, P.; Eckert, J.-F.; Nierengarten, J.-F. Int. J. Photoenergy 2001, 3, 33-40.

17. Watanabe, N.; Mizuno, T.; Matsumoto, M. Tetrahedron 2005, 61, 9569-9585.

18. Takaya, Y.; Terashima, K.; Ito, J.; He, Y.-H.; Tateoka, M.; Yamaguchi, N.; Niwa, M. Tetrahedron 2005, 61, 10285-10290.

19. Yasuda, M.; Yamasaki, S.; Onishi, Y.; Baba, A. Org. Synth. 2006, 83, 38-44.

20. Crosignani, S.; Nadal, B.; Li, Z.; Linclau, B. Chem. Comm. 2003 , 260-261.

21. Kim, K.-S.; Kwon, O. Z.; Seo, G. Stud. Surf. Sci. Catal. 2004, 154C, 2966-2972. 